\title{
Considerable escape of SARS-CoV-2 Omicron to antibody neutralization
}

https://doi.org/10.1038/s41586-021-04389-z

Received: 10 December 2021

Accepted: 23 December 2021

Published online: 23 December 2021

Check for updates

\author{
Delphine Planas ${ }^{1,2,15}$, Nell Saunders ${ }^{1,3,15}$, Piet Maes ${ }^{4,15}$, Florence Guivel-Benhassine', \\ Cyril Planchais ${ }^{5}$, Julian Buchrieser', William-Henry Bolland ${ }^{1,3}$, Françoise Porrot', \\ Isabelle Staropoli', Frederic Lemoine ${ }^{6}$, Hélène Pérée, ${ }^{7,8}$, David Veyer ${ }^{7,8}$, Julien Puech ${ }^{7}$, \\ Julien Rodary ${ }^{7}$, Guy Baele ${ }^{4}$, Simon Dellicour ${ }^{4,9}$, Joren Raymenants ${ }^{10}$, Sarah Gorissen ${ }^{10}$, \\ Caspar Geenen $^{10}$, Bert Vanmechelen ${ }^{4}$, Tony Wawina-Bokalanga ${ }^{4}$, Joan Martí-Carreras ${ }^{4}$, \\ Lize Cuypers ${ }^{11}$, Aymeric Sève ${ }^{12}$, Laurent Hocqueloux ${ }^{12}$, Thierry Prazuck ${ }^{12}$, Félix A. Rey ${ }^{13}$, \\ Etienne Simon-Loriere ${ }^{14}$, Timothée Bruel ${ }^{1,2,16} \bowtie$, Hugo Mouquet ${ }^{5,16 \bowtie}$, Emmanuel André $^{10,11,16 \bowtie}$ \& \\ Olivier Schwartz $z^{1,2,16 凶}$
}

\begin{abstract}
The SARS-CoV-2 Omicron variant was first identified in November 2021 in Botswana and South Africa $^{1-3}$. It has since spread to many countries and is expected to rapidly become dominant worldwide. The lineage is characterized by the presence of around 32 mutations in spike-located mostly in the $\mathrm{N}$-terminal domain and the receptor-binding domain-that may enhance viral fitness and enable antibody evasion. Here we isolated an infectious Omicron virus in Belgium from a traveller returning from Egypt. We examined its sensitivity to nine monoclonal antibodies that have been clinically approved or are in development ${ }^{4}$, and to antibodies present in 115 serum samples from COVID-19 vaccine recipients or individuals who have recovered from COVID-19. Omicron was completely or partially resistant to neutralization by all monoclonal antibodies tested. Sera from recipients of the Pfizer or AstraZeneca vaccine, sampled five months after complete vaccination, barely inhibited Omicron. Sera from COVID-19-convalescent patients collected 6 or 12 months after symptoms displayed low or no neutralizing activity against Omicron. Administration of a booster Pfizer dose as well as vaccination of previously infected individuals generated an anti-Omicron neutralizing response, with titres 6-fold to 23 -fold lower against Omicron compared with those against Delta. Thus, Omicron escapes most therapeutic monoclonal antibodies and, to a large extent, vaccine-elicited antibodies. However, Omicron is neutralized by antibodies generated by a booster vaccine dose.
\end{abstract}

In less than three weeks after its discovery, the Omicron variant was detected in dozens of countries. The WHO classified this lineage (previously known as Pango lineage B.1.1.529) as a variant of concern (VOC) on 26 November 2021 (ref. $^{1}$ ). Preliminary estimates of its doubling time range between 1.2 days and 3.6 days in populations with a high rate of SARS-CoV-2 immunity ${ }^{2,5}$. Omicron is expected to supplant the currently dominant Delta lineage during the next weeks or months. Little is known about its sensitivity to the humoral immune response. Recent reports indicated that Omicron has a reduced sensitivity to certain monoclonal and polyclonal antibodies ${ }^{6-10}$, and $\mathrm{CD}^{+} \mathrm{T}$ cell epitopes that were previously characterized in other variants seem to be conserved in Omicron ${ }^{11}$.

\section{Isolation and characterization of an Omicron variant}

We isolated an Omicron variant from a nasopharyngeal swab of an unvaccinated individual who developed moderate symptoms 11 days after returning to Belgium from Egypt. The virus was amplified by one passage in Vero E6 cells. The sequences of the swab and the outgrown virus were identical, and were identified as the Omicron variant (Pango lineage BA.1, GISAID:EPI_ISL_6794907 (swab) and EPI_ISL_7413964 (outgrown)) (Fig. 1a). The spike protein contained 32 changes compared with the D614G strain (belonging to the basal B.1 lineage), which we used here as a reference, including 7 changes in the $\mathrm{N}$-terminal domain (NTD), with substitutions, deletions and a 3 -amino-acid insertion (A67V, $\Delta 69-70$,

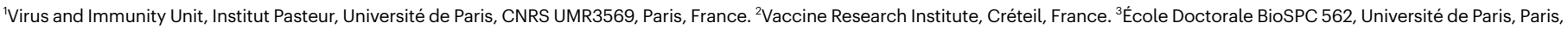
France. ${ }^{4}$ Department of Microbiology, Laboratory of Clinical and Epidemiological Virology, Immunology and Transplantation, Rega Institute, KU Leuven, Leuven, Belgium. ${ }^{5} \mathrm{Humoral}$

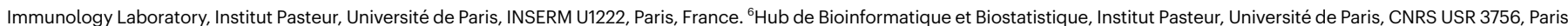

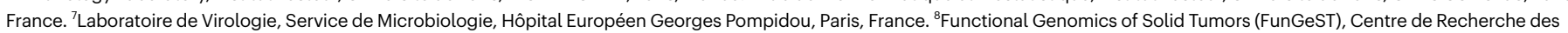
Cordelier, INSERM, Université de Paris, Sorbonne Université, Paris, France. ${ }^{9}$ Spatial Epidemiology Lab (SpELL), Université Libre de Bruxelles, Brussels, Belgium. ${ }^{10}$ Laboratory of Clinical Microbiology, Department of Microbiology, Immunology and Transplantation, KU Leuven, Leuven, Belgium. "Department of Laboratory Medicine, National Reference Centre for Respiratory Pathogens, University Hospitals Leuven, Leuven, Belgium. ${ }^{12}$ Service de Maladies Infectieuses, CHR d'Orléans, Orléans, France. ${ }^{13}$ Structural Virology Unit, Institut Pasteur, Université de Paris,

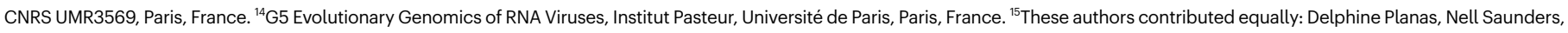
Piet Maes. ${ }^{16}$ These authors jointly supervised this work: Timothée Bruel, Hugo Mouquet, Emmanuel André, Olivier Schwartz. ${ }^{\bowtie}$-mail: timothee.bruel@pasteur.fr; hugo.mouquet@pasteur.fr; emmanuel.andre@uzleuven.be; olivier.schwartz@pasteur.fr 


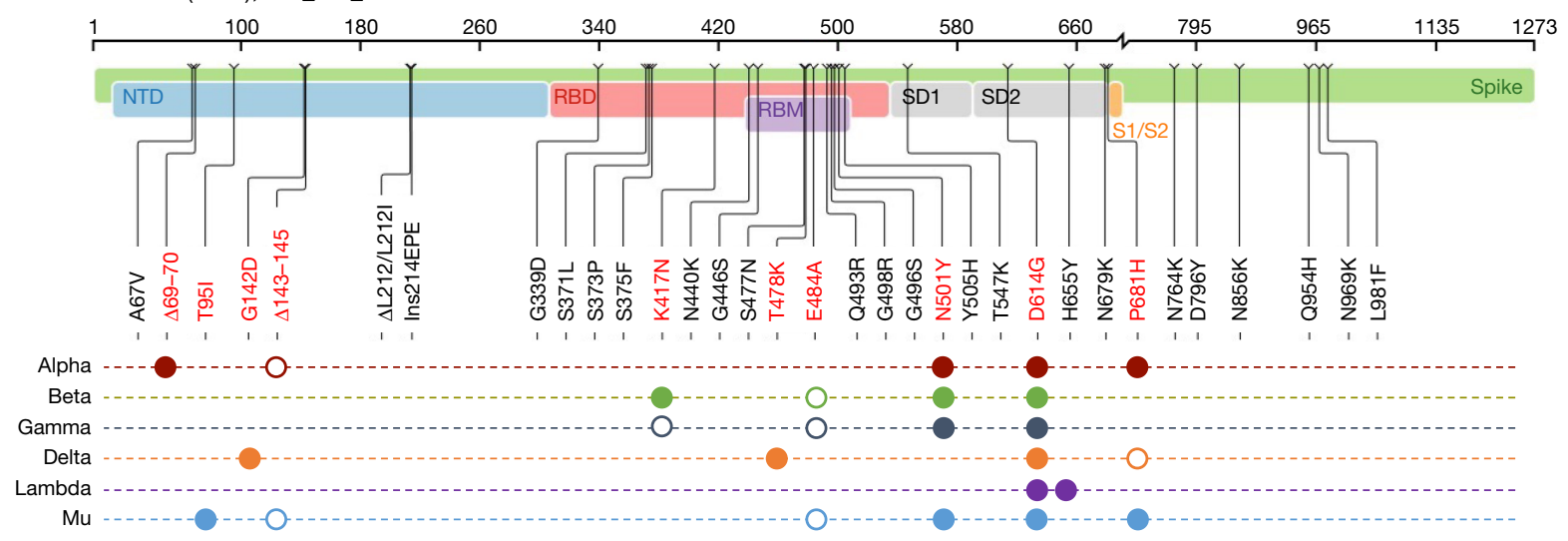

b

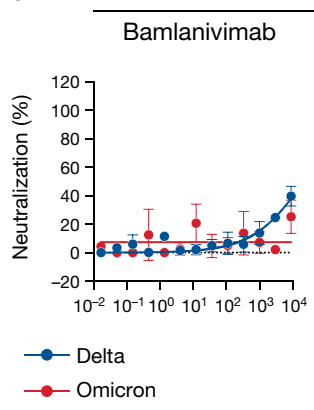

$\rightarrow$ Cilgavimab

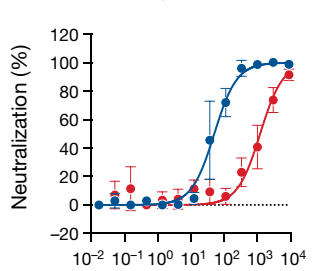

Lilly

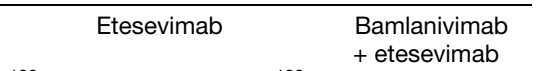

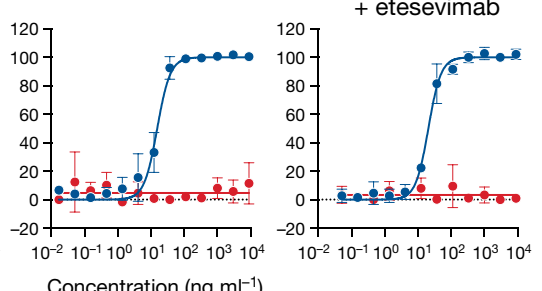

Concentration $\left(\mathrm{ng} \mathrm{ml}^{-1}\right)$

AstraZeneca

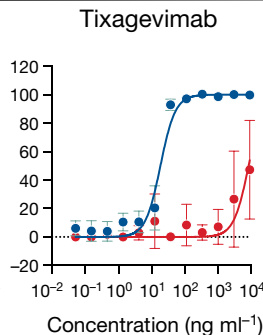

$\begin{aligned} & \text { Cilgavimab } \\ + & \text { tixagevimab }\end{aligned}$

Fig. 1 Neutralization of SARS-CoV-2 variants Delta and Omicron by clinical and preclinical monoclonal antibodies. a, The mutational landscape of the Omicron spike protein. The amino acid modifications are indicated in comparison to the ancestral Wuhan-Hu-1 sequence (GenBank:NC_045512). Consensus sequences of the spike protein were built using the Sierra tool ${ }^{38}$. The Omicron sequence corresponds to the viral strain that was isolated in Belgium and used in the study (GISAID:EPI_ISL_6794907). Mutations are compared to some pre-existing VOCs and variants of interest. The filled circles indicate changes identical to Omicron. The open circles indicate different substitutions at the same position. $\mathbf{b}$, Neutralization curves of monoclonal antibodies.

T95I, G142D, $\Delta 141-143, \Delta 211 \mathrm{~L} 212 \mathrm{I}$ and Ins214EPE), 15 mutations in the receptor-binding domain (RBD) (G339D, S371L, S373P, S375F, K417N, N440K, G446S, S477N, T478K, E484A, Q493R, G496S, Q498R and N501Y, and $\mathrm{Y} 505 \mathrm{H}$ ), the $\mathrm{T} 574 \mathrm{~K}$ mutation, 3 mutations close to the furin cleavage site (H655Y, N679K and $\mathrm{P} 681 \mathrm{H}$ ) and 6 mutations in the S2 region (N764K, D796Y, N856K, Q954H, N969 and L981F) (Fig. 1a). This extensive constellation of changes is unique, but includes at least 11 modifications that have been observed in other lineages and VOCs or at sites that are mutated in other variants (Fig. 1a). Viral stocks were titrated using S-Fuse reporter cells and Vero cells. S-Fuse cells become $\mathrm{GFP}^{+}$after infection, enabling rapid assessment of infectivity and the measurement of neutralizing antibody levels ${ }^{12-14}$. Syncytia were observed in Omicron-infected S-Fuse cells (Extended Data Fig. 1). Syncytia were smaller after infection with Omicron, relative to Delta (Extended Data Fig. 1). Future experiments will help to determine whether the fusogenic potential of Omicron is different from that of other variants ${ }^{15}$.
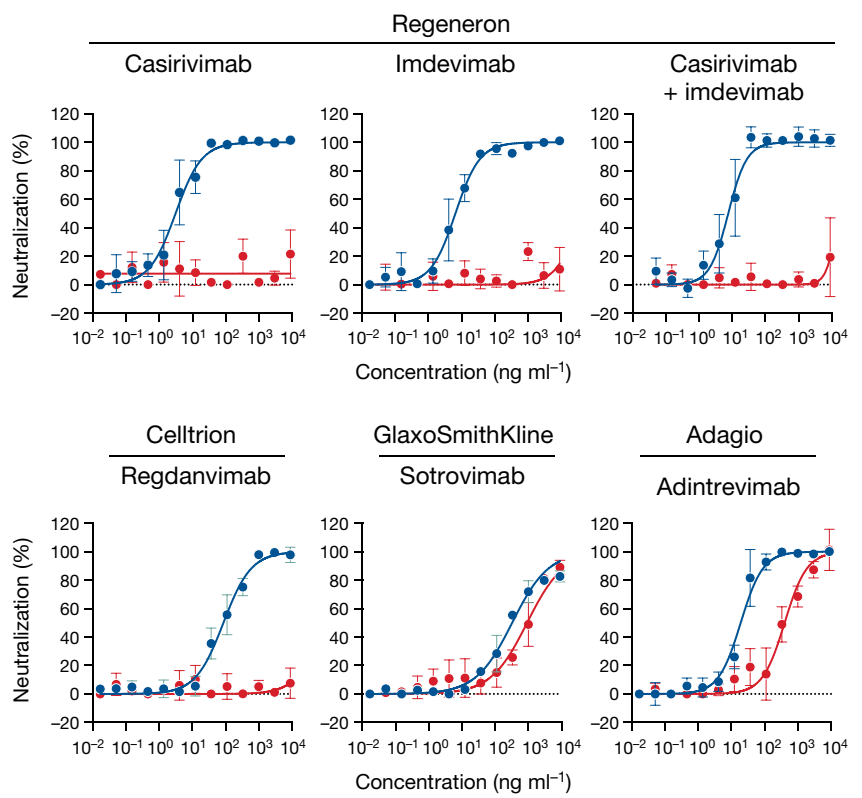

Dose-response analysis of the neutralization by clinical or preclinical monoclonal antibodies (bamlanivimab, etesevimab, casirivimab, imdevimab, adintrevimab, cilgavimab, tixagevimab, regdanvimab and sotrovimab) and the indicated combinations (bamlanivimab + etesevimab, casirivimab + imdevimab (corresponding to monoclonal antibodies present in Ronapreve),

cilgavimab + tixagevimab (corresponding to monoclonal antibodies present in Evusheld)) on Delta (blue dots) and Omicron (red dots) variants. Data are mean \pm s.d. of three independent experiments. The $\mathrm{IC}_{50}$ values for each antibody are presented in Extended Data Table1.

\section{Phylogenetic analysis of the Omicron lineage}

We inferred a global phylogeny by subsampling SARS-CoV-2 sequences that are available on the GISAID EpiCoV database. To better contextualize the isolated virus genome, we performed a focused phylogenetic analysis using all of the Omicron samples deposited on GISAID on 6 December 2021 as background (Extended Data Fig. 2). The tree topology indicates that the Omicron lineage does not directly derive from any of the previously described VOCs. The very long branch of the Omicron lineage in the time-calibrated tree (Extended Data Fig. 2) might reflect a cryptic and potentially complex evolutionary history. At the time of writing, no Omicron genomic sequences from Egypt were available on GISAID, nor do we know of any sequences of travellers that used the same planes. The isolated strain genome showed no close connection to other Belgian Omicron infections. Follow-up analyses with additional 
genomic data will improve the phylogenetic resolution to determine whether the patient was infected before or after returning to Belgium.

\section{The mutational landscape of Omicron}

We highlighted the 29 amino acid substitutions, the 3 amino-acid deletions and a 3-residue insertion that are present in the Omicron spike, with respect to the Wuhan strain, in a 3D model of the protein (Extended Data Fig. 3a). The 15 mutations in the RBD cluster around the trimer interface. The RBD is the target of the most potently neutralizing monoclonal antibodies against SARS-CoV-2, which have been divided into four classes depending of the location of their epitope ${ }^{4,16,17}$ (Extended Data Fig. 3b). Monoclonal antibodies in classes 1 and 2 compete for binding to human ACE2 (hACE2), whereas those from classes 3 and 4 bind away from the hACE2 interaction surface (Extended Data Fig. 3b). The epitopes of the class 2 and 3 monoclonal antibodies are exposed irrespective of the conformation of the RBD on the spike ('up' or 'down' configuration $^{18}$ ), whereas those of classes 1 and 4 require an RBD in the up conformation. Whereas the previous VOCs displayed mutations only in the region targeted by class 1 and 2 monoclonal antibodies, Omicron mutations are located within the epitopes of all four classes of monoclonal antibodies. The mutations, insertion and deletions in the NTD might also affect the recognition of this domain by antibodies.

\section{Neutralization of Omicron by monoclonal antibodies}

We next assessed the sensitivity of Omicron to a panel of human monoclonal antibodies using the S-Fuse assay. We tested nine antibodies that are in clinical use or in development ${ }^{19-25}$. These monoclonal antibodies belong to the four main classes of anti-RBD antibodies, ${ }^{4,16,17}$. Bamlanivimab and etesevimab (class 2 and class 1, respectively) are mixed in the Lilly cocktail. Casirivimab and imdevimab (class 1 and class 3, respectively) form the REGN-COV2 cocktail from Regeneron and Roche (Ronapreve). Cilgavimab and tixagevimab (class 2 and class 1 , respectively) from AstraZeneca are also used in combination (Evusheld). Regdanvimab (Regkirona; Celltrion) is a class 1 antibody. Sotrovimab (Xevudy) by GlaxoSmithKline and Vir Biotechnology is a class 3 antibody that displays activity against diverse coronaviruses. It targets an RBD epitope outside the receptor-binding motif that includes N343-linked glycans. Adintrevimab (ADG20), developed by Adagio, binds to an epitope that is located between the class 1 and class 4 sites.

We measured the activity of the nine antibodies described above against Omicron and included the Delta variant for comparison purposes (Fig.1b). As previously reported, bamlanivimab did not neutralize Delta $^{14,26,27}$. The other antibodies neutralized Delta with a 50\% inhibitory concentration $\left(\mathrm{IC}_{50}\right.$ ) varying from 3.1 to $325 \mathrm{ng} \mathrm{ml}^{-1}$ (Fig. $1 \mathrm{~b}$ and Extended Data Fig. 4). Five antibodies (bamlanivimab, etesevimab, casirivimab, imdevimab and regdanvimab) lost antiviral activity against Omicron. The four other antibodies displayed a 2 .8-fold to 453 -fold increase in IC $\mathrm{I}_{50}$ (ranging from 403 to $8,305 \mathrm{ng} \mathrm{ml}^{-1}$ ) against Omicron. Sotrovimab was the only antibody that displayed a rather similar activity against both strains, with an $\mathrm{IC}_{50}$ of 325 and $917 \mathrm{ng} \mathrm{ml}^{-1}$ against Delta and Omicron, respectively. We also tested the antibodies in combination to mimic the therapeutic cocktails. Bamlanivimab + etesevimab (Lilly) or casirivimab + imdevimab (Ronapreve) were inactive against Omicron. Cilgavimab + tixagevimab (Evusheld) neutralized Omicron with an $\mathrm{IC}_{50}$ of $773 \mathrm{ng} \mathrm{ml}^{-1}$, corresponding to a 58-fold increase relative to Delta (Fig. $1 \mathrm{~b}$ and Extended Data Fig. 4).

Next, using flow cytometry, we examined the binding of each monoclonal antibody to Vero cells infected with the Delta and Omicron variants (Extended Data Fig. 4). The five clinical antibodies that lost antiviral activity (bamlanivimab, etesevimab, casirivimab, imdevimab and regdanvimab) displayed a strong reduction (8-fold to 47 -fold and 11-fold to 242 -fold at 1 and $0.1 \mu \mathrm{g} \mathrm{ml}^{-1}$, respectively) in their binding to Omicron-infected cells compared with Delta-infected cells, as measured by the median fluorescence intensity of the signal (Extended Data Fig. 4). Cilgavimab, sotrovimab, tixagevimab and adintrevimab, which remained partly active, were less impaired in their binding to Omicron-infected cells (2-fold to 9-fold and 1.6-fold to 11-fold decrease at 1 and $0.1 \mu \mathrm{g} \mathrm{ml}^{-1}$, respectively) (Extended Data Fig. 4).

Thus, Omicron completely or partially escapes neutralization by the tested antibodies. Our results are consistent with findings reported in recent preprints ${ }^{7,8,10}$. The neutralization escape is correlated with a decrease in the binding of the antibodies to Omicron spike.

\section{Sensitivity of Omicron to sera from vaccinees}

We next examined whether vaccine-elicited antibodies neutralized Omicron. To achieve this, we randomly selected 54 individuals from a cohort established in the French city of Orléans, comprising vaccinated individuals who were not previously infected with SARS-CoV-2. The characteristics of the vaccinated individuals are shown in Extended Data Table1. Sixteen individuals received the Pfizer two-dose vaccine regimen and 18 received the AstraZeneca two-dose vaccine regimen. Twenty individuals who were vaccinated with the Pfizer vaccine received a booster dose. We measured the potency of the sera of these individuals against the Delta and Omicron strains. We used the D614G ancestral strain (belonging to the basal B.1 lineage) as a control (Fig. 2a). We calculated the $50 \%$ effective dilution $\left(\mathrm{ED}_{50}\right)$ for each combination of serum and virus. Sera were first sampled five months after the full two-dose vaccination. For the Pfizer vaccine, the levels of neutralizing antibodies were relatively low against D614G and Delta (median $\mathrm{ED}_{50}$ of neutralization of 329 and 91), reflecting the waning of the humoral response $^{14}$ (Fig. 2a). We did not detect any neutralization against the Omicron variant with these sera, except one, which displayed a low antiviral activity (Fig. 2a). The percentage of sera with detectable neutralizing activity is shown in Extended Data Fig. 5.

A similar pattern was observed for the AstraZeneca vaccine. Five months after vaccination, the levels of antibodies neutralizing Delta were low ( $\mathrm{ED}_{50}$ of 187 and 68 against D614G and Delta, respectively). No antiviral activity was detected against Omicron in $90 \%$ of the sera (Fig. 2a and Extended Data Fig. 5).

We next examined the effect of a Pfizer booster dose, administrated seven months after Pfizer vaccination. The sera were collected one month after the third dose. The booster dose enhanced neutralization titres against $\mathrm{D} 614 \mathrm{G}$ and Delta by 39 -fold and 49 -fold $\left(\mathrm{ED}_{50}\right.$ of 12,739 and 4,489 , respectively, compared with the sampling time 5 months after the full two-dose vaccination). The sera from 1 month after the booster dose were also associated with a strong increase in the neutralization activity against Omicron ( $\mathrm{ED}_{50}$ of 722 ) (Fig. $2 \mathrm{~b}$ ). At this time point, $100 \%$ of the tested sera displayed neutralizing activity (Extended Data Fig.5).

Taken together, these results indicate that Omicron is poorly or not neutralized by sera of vaccinated individuals sampled five months after vaccination. The booster dose triggered detectable cross-neutralization activity against Omicron. However, even after the booster dose, the variant displayed a decrease in $\mathrm{ED}_{50}$ of 18-fold and 6-fold compared with D614G and Delta, respectively.

\section{Sensitivity of Omicron to convalescent sera}

We subsequently examined the neutralization ability of sera from convalescent individuals. We randomly selected 61 longitudinal samples from 40 donors in a cohort of infected individuals from Orléans. Individuals were diagnosed with SARS-CoV-2 infection by quantitative PCR with reverse transcription (RT-qPCR) (Extended Data Table 1b). We previously studied the potency of these sera against D614G, Alpha, Beta and Delta isolates ${ }^{13,14}$. We analysed individuals sampled at a median of 6 and 12 months (M6 and M12) after the onset of symptoms. For the D614G and Delta variants, the neutralization titres were stable or slightly decreased over time (569 and 580 for D614G, and 235 and 143 


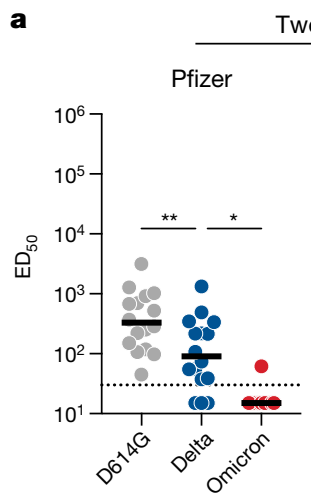

Two doses

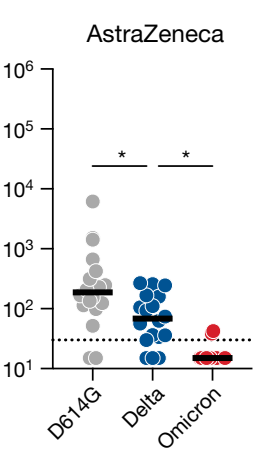

b

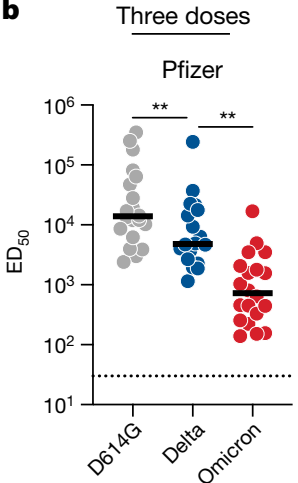

Fig. 2 | Sensitivity of the SARS-CoV-2 variants D614G, Delta and Omicron to sera from vaccinated, convalescent or infected-then-vaccinated

individuals. Neutralization titres of the sera against the three indicated viral variants are expressed as $\mathrm{ED}_{50}$. a, Neutralizing activity of sera from individuals who were vaccinated with the AstraZeneca ( $n=18$; left) and Pfizer ( $n=16$; right) vaccines, sampled at 5 months after the second dose.b, Neutralizing activity of sera from Pfizer-vaccinated recipients sampled one month (M1) after the third injection. $n=20$. The dotted line indicates the limit of detection $\left(\mathrm{ED}_{50}=30\right)$. c, The neutralizing activity of sera from convalescent individuals $(n=16)$,

for Delta, at M6 and M12, respectively) ${ }^{13}$ (Fig. 2c). The convalescent sera barely neutralized Omicron or did not inhibit Omicron at all at these time points. Only $36 \%$ and $39 \%$ of the samples displayed neutralizing activity against Omicron at M6 and M12, respectively, whereas the majority (91-94\%) were active against Delta (Extended Data Fig. 5).

Twenty-two individuals were vaccinated at M12 with a Pfizer dose. Sera sampled 1 month after vaccination showed a considerable increase in neutralizing antibody titres against the D614G and Delta variants, reaching a median $\mathrm{ED}_{50}$ of 78,162 and 33,536, respectively (Fig. 2d). These sera also neutralized Omicron, with a median $\mathrm{ED}_{50}$ of 1,466 (Fig. 2d). Thus, as shown for other variants ${ }^{13,28,29}$, a single dose of vaccine boosted cross-neutralizing antibody responses to Omicron in previously infected individuals. However, the neutralization titres were reduced by 53 -fold and 23-fold compared with D614G and Delta, respectively.

\section{Discussion}

Omicron has opened a new chapter in the COVID-19 pandemic ${ }^{2,30}$. The principal concerns about this variant include its high transmissibility, as underlined by its rapid spread in different countries, and the presence of more than 55 mutations spanning the whole viral genome. Omicron contains 32 mutations in its spike protein in the NTD, RBD and in vicinity of the furin cleavage site. Some mutations were already present in other VOCs and variants of interest, and have been extensively characterized $^{30-32}$. Owing to their position, they are expected to affect the binding of natural or therapeutic antibodies, to increase affinity to ACE2 and to enhance the fusogenic activity of the spike. Future work will help to determine how this association of mutations affects viral fitness in culture systems and their contribution to the high transmissibility of the variant.

Here we studied the cross-reactivity of clinical or preclinical monoclonal antibodies, as well as of $\mathbf{1 1 5}$ sera from vaccine recipients and long-term convalescent individuals against an infectious Omicron isolate. We report that, among nine monoclonal antibodies in clinical use or in development, six (bamlanivimab, etesevimab, casirivimab, imdevimab, tixagevimab and regdanvimab) were inactive against Omicron. Two other antibodies (cilgavimab and adintrevimab) displayed about a 20 -fold increase in $\mathrm{IC}_{50}$. Sotrovimab was less affected by Omicron's mutations, with an increase in $\mathrm{IC}_{50}$ by only threefold. Wealso show that Omicron was barely neutralized by sera from vaccinated c

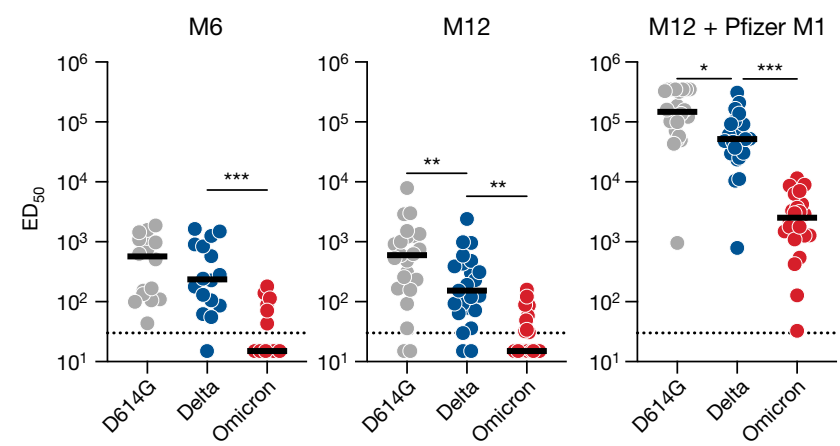

sampled at 6 months after the onset of symptoms (left). Middle, the neutralizing activity of sera from convalescent individuals $(n=23)$, sampled at 12 months after the onset of symptoms. Right, the neutralizing activity of sera from individuals who were infected then vaccinated $(n=22)$, sampled one month after the first injection (right). In each panel, data are the mean values from two to three independent experiments. Two-sided Friedman tests with Dunn's multiple-comparison correction was performed to compare D614G and Omicron with the Delta variant; ${ }^{*} P<0.05,{ }^{* *} P<0.01,{ }^{* * *} P<0.001$.

individuals who were sampled 5 months after the administration of two doses of the Pfizer or AstraZeneca vaccine. Sera from convalescent individuals at 6 or 12 months after infection barely neutralized or did not detectably neutralize Omicron.

The decrease in antibody efficacy helps to explain the high number of breakthrough infections and reinfection cases, and the spread of Omicron in both non-immune and immune individuals ${ }^{33}$. There is currently no evidence of increased disease severity associated with Omicron compared with Delta, either among naive or immunized individuals. It is probable that, even if pre-existing SARS-CoV-2 antibodies may poorly prevent Omicron infection, anamnestic responses and cellular immunity will be operative to prevent severe forms of the disease ${ }^{34}$.

We further report that a booster dose of Pfizer vaccine, as well as vaccination of previously infected individuals, strongly increased overall levels of anti-SARS-CoV-2 neutralizing antibodies, well above a threshold allowing inhibition of Omicron. Affinity maturation of antibodies is known to improve the efficacy of the humoral anti-SARS-CoV-2 response over time $\mathrm{e}^{35,36}$. This process helps to explain the efficacy of booster doses. However, sera with high antibody levels displayed a 6-fold to 23-fold reduction in neutralization efficacy against Omicron compared with the currently predominant Delta strain.

Potential limitations of our work include the low number of sera analysed from vaccine recipients and convalescent individuals, and the lack of characterization of cellular immunity, which is known to be more cross-reactive than the humoral response. Our results may therefore partly underestimate the residual protection offered by vaccines and previous infections against Omicron infection, in particular with regard to the severity of disease. We analysed only sera sampled one month after the booster dose, or after vaccination of infected individuals. Future work with more individuals and longer survey periods will help to characterize the duration of the humoral response against Omicron. We focused on immune responses elicited by Pfizer and AstraZeneca vaccination. It will be worth determining the potency of other vaccines against this variant.

We focused our analyses on one single viral isolate, corresponding to the archetype Omicron variant sequence (Pango BA.1 lineage). Two related lineages with additional mutations (BA.2 and BA.3) have recently emerged and are less widely spread. It will be worth comparing the behaviour of viral isolates from these more recent lineages with the main BA.1 Omicron strain. 
Our results have important public health consequences regarding the use of therapeutic monoclonal antibodies and vaccines. Clinical indications of monoclonal antibodies include pre-exposure prophylaxis in individuals who are unable to mount an immune response, as well as the prevention of COVID-19 in infected individuals who are at high risk for progressing towards severe disease. Antibody-based treatment strategies need to be rapidly adapted to Omicron. Experiments in preclinical models or clinical trials are warranted to assess whether the drops in $\mathrm{IC}_{50}$ are translated into impaired clinical efficacy of the monoclonal antibodies that retain efficacy against Omicron. Most low-income countries display a low vaccination rate, a situation that probably facilitates SARS-CoV-2 spread and continuous evolution. A booster dose improves the quality and the level of the humoral immune response, and is associated with a strong protection against severe forms of the disease $\mathrm{e}^{37}$. An accelerated deployment of vaccines and boosters throughout the world is necessary to counteract viral spread. Our results also suggest that there is a need to update and complete the current pharmacopoeia, in particular with regard to vaccines and monoclonal antibodies.

\section{Online content}

Any methods, additional references, Nature Research reporting summaries, source data, extended data, supplementary information, acknowledgements, peer review information; details of author contributions and competing interests; and statements of data and code availability are available at https://doi.org/10.1038/s41586-021-04389-z.

1. Classification of Omicron (B.1.1.529): SARS-CoV-2 Variant of Concern, https://www.who.int/ news/item/26-11-2021-classification-of-omicron-(b.1.1.529)-sars-cov-2-variant-of-concern (WHO, 2021)

2. Karim, S. S. A. \& Karim, Q. A. Omicron SARS-CoV-2 variant: a new chapter in the COVID-19 pandemic. Lancet 398, 2126-2128 (2021).

3. Viana, R. et al. Rapid epidemic expansion of the SARS-CoV-2 Omicron variant in southern Africa. Nature, https://doi.org/10.1038/s41586-022-04411-y (2022).

4. Taylor, P. C. et al. Neutralizing monoclonal antibodies for treatment of COVID-19. Nat. Rev. Immunol. 21, 382-393 (2021).

5. Grabowski, F., Kochańczyk, M. \& Lipniacki, T. Omicron strain spreads with the doubling time of 3.2-3.6 days in South Africa province of Gauteng that achieved herd immunity to Delta variant. Preprint at https://doi.org/10.1101/2021.12.08.21267494 (2021).

6. Cele, S. et al. Omicron extensively but incompletely escapes Pfizer BNT162b2 neutralization. Nature, https://doi.org/10.1038/s41586-021-04387-1 (2021).

7. Cao, Y. et al. Omicron escapes the majority of existing SARS-CoV-2 neutralizing antibodies. Nature, https://doi.org/10.1038/s41586-021-04385-3 (2021).

8. Cameroni, E. et al. Broadly neutralizing antibodies overcome SARS-CoV-2 Omicron antigenic shift. Nature, https://doi.org/10.1038/s41586-021-04386-2 (2021).

9. Dejnirattisai, W. et al. Reduced neutralisation of SARS-COV-2 Omicron-B.1.1.529 variant by post-immunisation serum. Lancet 399, 234-236 (2021).

10. Aggarwal A et al SARS-CoV-2 Omicron: evasion of potent humoral responses and resistance to clinical immunotherapeutics relative to viral variants of concern. Preprint at https://doi.org/10.1101/2021.12.14.21267772 (2021)

11. Redd, A. D. et al. Minimal cross-over between mutations associated with Omicron variant of SARS-CoV-2 and $\mathrm{CD}^{+}{ }^{+} \mathrm{T}$ cell epitopes identified in COVID-19 convalescent individuals. Preprint at https://doi.org/10.1101/2021.12.06.471446 (2021).
12. Buchrieser, J. et al. Syncytia formation by SARS-CoV-2 infected cells. EMBO J. 39 e106267 (2020).

13. Planas, D. et al. Sensitivity of infectious SARS-CoV-2 B.1.1.7 and B.1.351 variants to neutralizing antibodies. Nat. Med. 27, 917-924 (2021).

14. Planas, D. et al. Reduced sensitivity of SARS-CoV-2 variant Delta to antibody neutralization. Nature 596, 276-280 (2021).

15. Rajah, M. M. et al. SARS-CoV-2 Alpha, Beta, and Delta variants display enhanced spike-mediated syncytia formation. EMBO J. 40, e108944 (2021).

16. Liu, L. et al. Potent neutralizing antibodies against multiple epitopes on SARS-CoV-2 spike. Nature 584, 450-456 (2020)

17. Barnes, C. O. et al. SARS-CoV-2 neutralizing antibody structures inform therapeutic strategies. Nature 588, 682-687 (2020).

18. Wrapp, D. et al. Cryo-EM structure of the 2019-nCoV spike in the prefusion conformation Science 367, 1260-1263 (2020).

19. Rappazzo, C. G. et al. Broad and potent activity against SARS-like viruses by an engineered human monoclonal antibody. Science 371, 823-829 (2021).

20. Zost, S. J. et al. Potently neutralizing and protective human antibodies against SARS-CoV-2. Nature 584, 443-449 (2020).

21. Kim, C. et al. A therapeutic neutralizing antibody targeting receptor binding domain of SARS-CoV-2 spike protein. Nat. Commun. 12, 288 (2021).

22. Shi, R. et al. A human neutralizing antibody targets the receptor-binding site of SARS-CoV-2. Nature 584, 120-124 (2020).

23. Jones, B. E. et al. The neutralizing antibody, LY-CoV555, protects against SARS-CoV-2 infection in nonhuman primates. Sci. Transl. Med. 13, eabf1906 (2021).

24. Hansen, J. et al. Studies in humanized mice and convalescent humans yield a SARS-CoV-2 antibody cocktail. Science 369, 1010-1014 (2020).

25. Pinto, D. et al. Cross-neutralization of SARS-CoV-2 by a human monoclonal SARS-CoV antibody. Nature 583, 290-295 (2020)

26. Liu, J. et al. BNT162b2-elicited neutralization of B.1.617 and other SARS-CoV-2 variants. Nature 596, 273-275 (2021)

27. Lucas, C. et al. Impact of circulating SARS-CoV-2 variants on mRNA vaccine-induced immunity. Nature 600, 523-529 (2021).

28. Gallais, F. et al. Evolution of antibody responses up to 13 months after SARS-CoV-2 infection and risk of reinfection,EBioMedicine 71, 103561 (2021).

29. Krammer, F. et al. Antibody responses in seropositive persons after a single dose of SARS-CoV-2 mRNA vaccine. New Engl. J. Med. 384, 1372-1374 (2021).

30. McCallum, M. et al. SARS-CoV-2 immune evasion by the B.1.427/B.1.429 variant of concern. Science 373, 648-654 (2021)

31. Plante, J. A. et al. The variant gambit: COVID-19's next move. Cell Host Microbe 29 508-515 (2021).

32. Starr, T. N. et al. Prospective mapping of viral mutations that escape antibodies used to treat COVID-19. Science 371, 850-854 (2021)

33. Pulliam, J. R. C. et al. Increased risk of SARS-CoV-2 reinfection associated with emergence of the Omicron variant in South Africa. Preprint at https://doi.org/ 10.1101/2021.11.11.21266068 (2021).

34. Gagne, M. et al. Protection from SARS-CoV-2 Delta one year after mRNA-1273 vaccination in rhesus macaques is coincident with anamnestic antibody response in the lung. Cell 185, 113-130 (2021).

35. Gaebler, C. et al. Evolution of antibody immunity to SARS-CoV-2. Nature 591, 639-644 (2021).

36. Wang, Z. et al. Naturally enhanced neutralizing breadth against SARS-CoV-2 one year after infection. Nature 595, 426-431 (2021).

37. Barda, N. et al. Effectiveness of a third dose of the BNT162b2 mRNA COVID-19 vaccine for preventing severe outcomes in Israel: an observational study. Lancet 398, 2093-2100 (2021).

38. Tzou, P. L. et al. Coronavirus Antiviral Research Database (CoV-RDB): an online database designed to facilitate comparisons between candidate anti-coronavirus compounds. Viruses 12, 1006 (2020)

Publisher's note Springer Nature remains neutral with regard to jurisdictional claims in published maps and institutional affiliations.

(c) The Author(s), under exclusive licence to Springer Nature Limited 2021 


\section{Methods}

No statistical methods were used to predetermine sample size. The experiments were not randomized and the investigators were not blinded to allocation during experiments and outcome assessment. Our research complies with all relevant ethical regulation.

\section{Orléans Cohort of convalescent and vaccinated individuals}

Since 27 August 2020, a prospective, monocentric, longitudinal, interventional cohort clinical study enrolling 170 individuals infected with SARS-CoV-2 with different disease severities, and 59 non-infected healthy control individuals is ongoing, aiming to describe the persistence of specific and neutralizing antibodies over a 24-month period. This study was approved by the ILE DE FRANCE IV ethical committee. At enrolment, written informed consent was collected and the participants completed a questionnaire that covered sociodemographic characteristics, virological findings (SARS-CoV-2 RT-PCR results, including date of testing), clinical data (date of symptoms onset, type of symptoms and hospitalization), and data related to anti-SARS-CoV-2 vaccination if ever (brand product, and date of first and second doses). The serological status of the participants was assessed every three months. Those who underwent anti-SARS-CoV-2 vaccination had regular blood sampling after first dose of vaccine (ClinicalTrials.gov: NCT04750720). The primary outcome was the presence of antibodies against the SARS-CoV-2 spike protein, as measured using the S-Flow assay. The secondary outcome was the presence of neutralizing antibodies as measured using the S-Fuse assay. For this study, we selected 61 convalescent and 54 vaccinated participants. Some individuals were sampled multiple times. We analysed a total of 115 sera. Study participants did not receive any compensation. The characteristics of each individual from the two cohorts are presented in Supplementary Table 2. The cohorts were constituted before the occurrence of the Omicron variant.

\section{Phylogenetic analysis}

To contextualize the isolated Omicron genome, all SARS-CoV-2 sequences available on the GISAID EpiCov database as of 6 December 2021 were retrieved. A subset of complete and high-coverage sequences, as indicated in GISAID, assigned to lineages B.1.529 or BA.1 and BA.2, were randomly subsampled. This subset was included in a global SARS-CoV-2 phylogeny reconstructed with augur and visualized with auspice as implemented in the Nextstrain pipeline (https:// github.com/nextstrain/ncov, version from 6 May 2021) ${ }^{39}$. Within Nextstrain, a random subsampling approach capping a maximum number of sequences per global region was used. The acknowledgment of contributing and originating laboratories for all sequences used in the analysis is provided in Supplementary Table 1.

\section{D representation of mutations on the surface of spike of B1.617.2 and other variants}

The panels in Extended Data Fig. 3 were prepared using The PyMOL Molecular Graphics System, v.2.1 (Schrödinger). The atomic model used (Protein Data Bank: 6XR8) has previously been described ${ }^{40}$.

\section{S-Fuse neutralization assay}

U2OS-ACE2 GFP1-10 or GFP 11 cells, also termed S-Fuse cells, become $\mathrm{GFP}^{+}$when they are productively infected by SARS-CoV-2 (refs. ${ }^{12,13}$ ). Cells tested negative for mycoplasma. Cells were mixed (ratio 1:1) and plated at $8 \times 10^{3}$ per well in a $\mu$ Clear 96-well plate (Greiner Bio-One). The indicated SARS-CoV-2 strains were incubated with serially diluted monoclonal antibodies or sera for $15 \mathrm{~min}$ at room temperature and added to S-Fuse cells. The sera were heat-inactivated for $30 \mathrm{~min}$ at $56{ }^{\circ} \mathrm{C}$ before use. Then, $18 \mathrm{~h}$ later, cells were fixed with $2 \% \mathrm{PFA}$, washed and stained with Hoechst (dilution of 1:1,000, Invitrogen). Images were acquired using an Opera Phenix high-content confocal microscope (PerkinElmer). The GFP area and the number of nuclei were quantified using the Harmony software (PerkinElmer). The percentage of neutralization was calculated using the number of syncytia as value with the following formula: $100 \times(1$ - (value with serum - value in 'non-infected')/ (value in 'no serum' - value in 'non-infected')). Neutralizing activity of each serum was expressed as the half maximal effective dilution $\left(E_{50}\right) . E_{50}$ values (in $\mu \mathrm{g} \mathrm{ml}^{-1}$ for monoclonal antibodies and in dilution values for sera) were calculated with a reconstructed curve using the percentage of the neutralization at the different concentrations.

\section{Characteristics of the patient infected with Omicron}

The 32-year-old woman was unvaccinated and developed moderate symptoms on 22 November 2021, 11 days after returning to Belgium from Egypt via Turkey (stop-over to switch flights, without having left the airport). She did not display any risk factor for severe COVID-19 and rapidly recovered. She transmitted the virus to her husband but not to their children. She provided informed written consent to use the swab for future studies. The nasopharyngeal swab tested positive for SARS-CoV-2 on this date. The leftover material of the sample was used in this study after performing routine diagnostics, within the context of the mandate that was provided to UZ/KU Leuven as National Reference Center (NRC) of respiratory pathogens, as described in detail in the Belgian Royal Decree of 09/02/2011.

\section{Virus strains}

The reference D614G strain (hCoV-19/France/GE1973/2020) was supplied by the National Reference Centre for Respiratory Viruses hosted by Institut Pasteur and headed by S. van der Werf. This viral strain was supplied through the European Virus Archive goes Global (Evag) platform, a project that has received funding from the European Union's Horizon 2020 research and innovation program under grant agreement no 653316. The variant strains were isolated from nasal swabs using Vero E6 cells and amplified by one or two passages. Delta was isolated from a nasopharyngeal swab of a hospitalized patient returning from India ${ }^{14}$. The swab was provided and sequenced by the laboratory of Virology of Hopital Européen Georges Pompidou (Assistance Publique, Hopitaux de Paris). The Omicron-positive sample was cultured on Vero E6 cells as previously described ${ }^{41}$. Viral growth was confirmed by RT-qPCR three days after infection. At day 6 after infection, a cytopathic effect was detected and a full-length sequencing of the virus was performed. The Omicron strain was supplied and sequenced by the NRC UZ/KU Leuven. Both patients provided informed consent for the use of the biological materials. Titration of viral stocks was performed on Vero E6 cells, with a limiting dilution technique enabling the calculation of the median tissue culture infectious dose, or on S-Fuse cells. Viruses were sequenced directly on nasal swabs, and after one or two passages on Vero cells. The sequences were deposited on GISAID immediately after their generation (D614G:EPI_ISL_414631; Delta ID:EPI_ISL_2029113; Omicron ID:EPI_ISL_6794907.

\section{Flow cytometry}

Vero cells were infected with the indicated viral strains at a multiplicity of infection of 0.01. Two days after, cells were detached using PBS-0.1\% EDTA and transferred into U-bottom 96 -well plates $(50,000$ cells per well). Cells were then incubated for $30 \mathrm{~min}$ at $4{ }^{\circ} \mathrm{C}$ with the indicated monoclonal antibodies (1 or $0.1 \mu \mathrm{g} \mathrm{ml}^{-1}$ ) in MACS buffer (PBS, $5 \mathrm{~g} \mathrm{l}^{-1} \mathrm{BSA}$, 2 mM EDTA). Cells were washed with PBS and stained using anti-IgG AF647 (1:600 dilution in MACS buffer) (Thermo Fisher Scientific). Stainings were also performed on control non-infected cells. Cells were then fixed in 4\% PFA for 30 min at room temperature. Data were acquired on the Attune Nxt instrument using Attune Nxt Software v.3.2.1 (Life Technologies) and analysed using FlowJo v.10.7.1 (Becton Dickinson).

\section{Antibodies}

Four clinically available antibodies (bamlanivimab, casirivimab, etesevimab and imdevimab) were provided by CHR Orleans. 
The other human SARS-CoV-2 anti-RBD neutralizing antibodies (ADG20 or adintrevimab, AZD1061 (COV2-2130) or cilgavimab, AZD8895(COV22196) or tixagevimab, CT-P59 or regdanvimab, LY-CoV016 (CB6) or etesevimab, LY-CoV555 or bamlanivimab, REGN10933 or casirivimab, REGN10987 or imdevimab, and VIR-7831 (S309) or sotrovimab ${ }^{19-25}$ were produced as follows. DNA fragments encoding their IgH and IgL variable domains were synthetized (Life Technologies, Thermo Fisher Scientific). Purified digested DNA fragments were cloned into human Ig $\gamma 1$ - and IgK-/Ig $\lambda$-expressing vectors ${ }^{42}$ and recombinant IgG1 antibodies were produced by transient co-transfection of Freestyle 293-F suspension cells (Thermo Fisher Scientific) using the PEI-precipitation method as previously described ${ }^{43}$. IgG1 antibodies were purified by batch/gravity-flow affinity chromatography using protein G Sepharose 4 Fast Flow Beads (Cytivia) according to the manufacturer's instructions, dialysed against PBS using Slide-A-Lyzer dialysis cassettes (Thermo Fisher Scientific), quantified using the NanoDrop 2000 instrument (Thermo Fisher Scientific), and checked for purity and quality on a silver-stained SDS-PAGE gel (3-8\% Tris-Acetate Novex, Thermo Fisher Scientific). The pan-coronavirus anti-S2 non-neutralizing antibody Ab-10 was previously described ${ }^{13,14}$.

\section{Statistical analysis}

Flow cytometry data were analysed using FlowJo v.10 (TriStar). Calculations were performed using Excel 365 (Microsoft). Figures were generated using Prism 9 (GraphPad Software). Statistical analysis was conducted using GraphPad Prism 9. Statistical significance between different groups was calculated using the tests indicated in each figure legend.

\section{Reporting summary}

Further information on research design is available in the Nature Research Reporting Summary linked to this paper.

\section{Data availability}

All data supporting the findings of this study are available within the Article or from the corresponding authors on request. Viral sequences are available on request and were deposited at GISAID (https://www. gisaid.org/) under the following numbers: D614G (EPI_ISL_414631), Delta ID (EPI_ISL_2029113) and Omicron ID (EPI_ISL_6794907). Source data are provided with this paper.

39. Hadfield, J. et al. Nextstrain: real-time tracking of pathogen evolution. Bioinformatics $\mathbf{3 4}$ 4121-4123 (2018)

40. Cai, Y. et al. Distinct conformational states of SARS-CoV-2 spike protein. Science $\mathbf{3 6 9}$ 1586-1592 (2020).
41. Van Cleemput, J. et al. Organ-specific genome diversity of replication-competent SARS-CoV-2. Nat. Commun. 12, 6612 (2021)

42. Tiller, T. et al. Efficient generation of monoclonal antibodies from single human B cells by single cell RT-PCR and expression vector cloning. J. Immunol. Methods 329, 112-124 (2008).

43. Lorin, V. \& Mouquet, H. Efficient generation of human IgA monoclonal antibodies. J. Immunol. Methods 422, 102-110 (2015).

44. Walls, A. C. et al. Structure, function, and antigenicity of the SARS-CoV-2 spike glycoprotein. Cell 181, 281-292 (2020).

Acknowledgements We thank the staff at the European Health Emergency Preparedness and Response Authority (HERA) for their support. S. Cole for his help in initiating the collaboration between Institut Pasteur and KU Leuven through the HERA network; A. Baidaliuk and F. Gambaro for their help with the sequencing data analysis; the patients who participated to this study; the members of the Virus and Immunity Unit and other teams for discussions and help; N. Aulner and the staff at the UtechS Photonic Biolmaging (UPBI) core facility (Institut Pasteur)-a member of the France Biolmaging network-for image acquisition and analysis; the members of the KU Leuven University authorities, and J. Arnout, B. Lambrecht, C. Van Geet and L. Sels for their support: L. Belec, N. Robillard and M. Saliba for their help with sequencing; and F. Peira, V. Legros and L. Courtellemont for their help with the cohorts. The Opera system was co-funded by Institut Pasteur and the Région ile de France (DIM1Health). Work in the O.S laboratory is funded by Institut Pasteur, Urgence COVID-19 Fundraising Campaign of Institut Pasteur, Fondation pour la Recherche Médicale (FRM), ANRS, the Vaccine Research Institute (ANR-10-LABX-77), Labex IBEID (ANR-10-LABX-62-IBEID), ANR/FRM Flash Covid PROTEO-SARS-CoV-2 and IDISCOVR. Work in the UPBI is funded by grant ANR-10-INSB-04-01 and Région lle-de-France program DIM1-Health. D.P. is supported by the Vaccine Research Institute. The H.M. laboratory is funded by the Institut Pasteur, the Milieu Interieur Program (ANR-10-LABX-69-01), the INSERM, REACTing, EU (RECOVER) and Fondation de France (00106077) grants. The E.S.-L. laboratory is funded by Institut Pasteur, the INCEPTION program (Investissements d'Avenir grant ANR-16-CONV-0005) and the French Government's Investissement d'Avenir programme, Laboratoire d'Excellence 'Integrative Biology of Emerging Infectious Diseases' (grant no. ANR-10-LABX-62-IBEID). G.B. acknowledges support from the Internal Funds KU Leuven under grant agreement C14/18/094, and the Research Foundation-Flanders (Fonds voor Wetenschappelijk Onderzoek-Vlaanderen, GOE142ON, G098321N). P.M. acknowledges support from a COVID-19 research grant of 'Fonds Wetenschappelijk Onderzoek'/Research Foundation-Flanders (grant no. GOH442ON). S.D. is supported by the Fonds National de la Recherche Scientifique (FNRS, Belgium) and also acknowledges support from the Research Foundation-Flanders (Fonds voor Wetenschappelijk Onderzoek-Vlaanderen, G098321N) and from the European Union Horizon 2020 project MOOD (grant no. 874850). The funders of this study had no role in study design, data collection, analysis and interpretation, or writing of the article.

Author contributions Experimental strategy design, experiments: D.P., N.S., F.G.-B., C.P., J.B., W.-H.B., F.P., I.S., F.A.R., E.S.-L., T.B., H.M. and O.S. Vital materials: P.M., C.P., F.L., H.P., D.V., J.P., J. Rodary, G.B., S.D., J. Raymenants, S.G., C.G., B.V., T.W.-B., J.M.-C., L.C., A.S., L.H., T.P., H.M. and E.A. Phylogenetic analysis: G.B., E.S.-L., F.L. and S.D. Manuscript writing: D.P., F.A.R., E.S.-L., T.B., H.M., E.A. and O.S. Manuscript editing: D.P., N.S., P.M., G.B., L.C., F.A.R., E.S.-L., T.B., H.M., E.A. and O.S.

Competing interests C.P., H.M., O.S, T.B. and F.A.R. have a pending patent application for an anti-RBD monoclonal antibody that was not used in this study (PCT/FR2021/070522).

\section{Additional information}

Supplementary information The online version contains supplementary material available at https://doi.org/10.1038/s41586-021-04389-z.

Correspondence and requests for materials should be addressed to Timothée Bruel, Hugo Mouquet, Emmanuel André or Olivier Schwartz.

Peer review information Nature thanks the anonymous reviewers for their contribution to the peer review of this work.

Reprints and permissions information is available at http://www.nature.com/reprints. 


\section{Article}

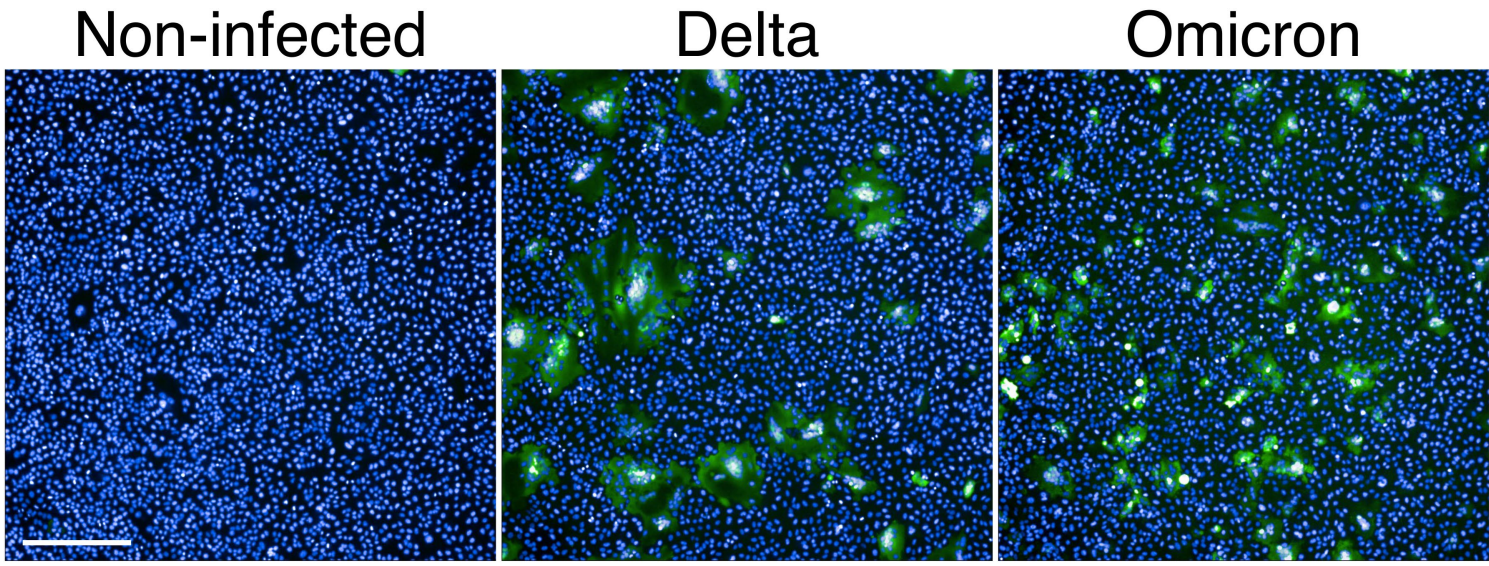

Extended Data Fig. 1|SARS-CoV-2 variants induce syncytia in S-Fuse cells. $\mathrm{S}$-Fuse cells were exposed to the indicated SARS-CoV-2 strains, at a multiplicity of infection (MOI) of $10^{-3}$. The cells become GFP+ when they fuse together.

After $20 \mathrm{~h}$, infected cells were stained with Hoechst to visualize nuclei. Syncytia (green) and nuclei (blue) are shown. Representative images from three independent experiments are shown. Scale bar, $500 \mu \mathrm{m}$. 


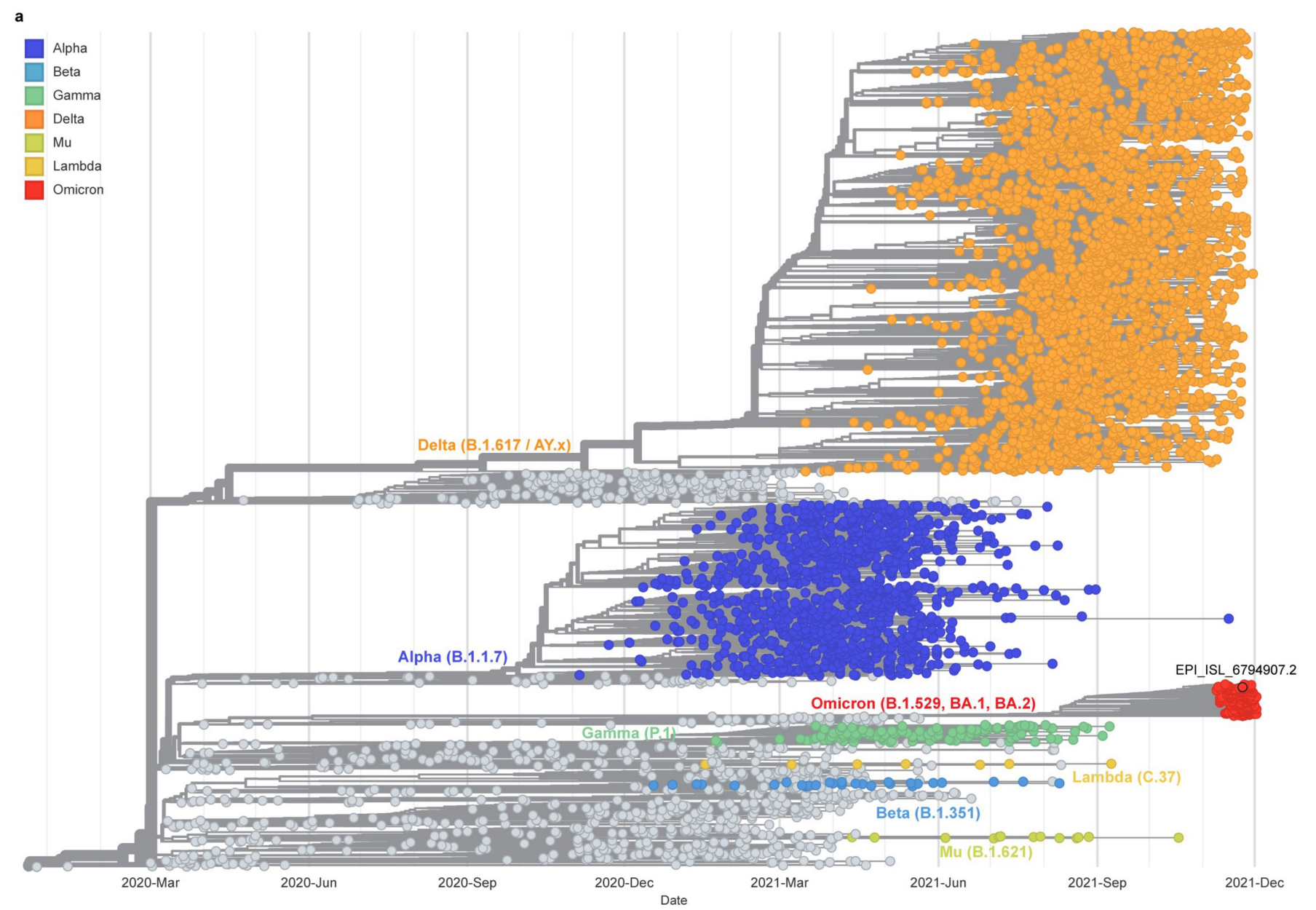

Extended Data Fig. 2 | Global phylogeny of SARS-CoV-2 highlighting the Omicron lineage. Time calibrated global SARS-CoV-2 phylogeny available from the Nextstrain platform (https://nextstrain.org/ncov/gisaid/global) ${ }^{39}$.
The position of the isolated Omicron variant is highlighted, and the variants of concern (VOCs) (Alpha, Beta, Gamma, Delta and Omicron) and variants of interest (VOIs) (Lambda, Mu) are coloured as indicated. 


\section{Article}

a.

Top view

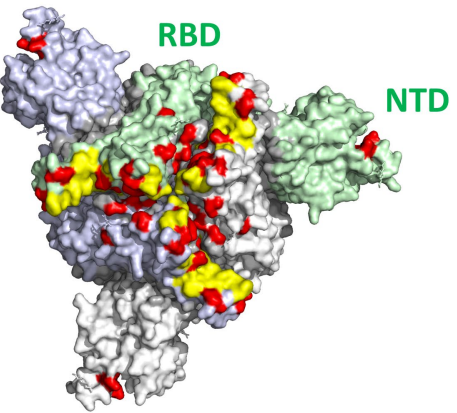

Side view

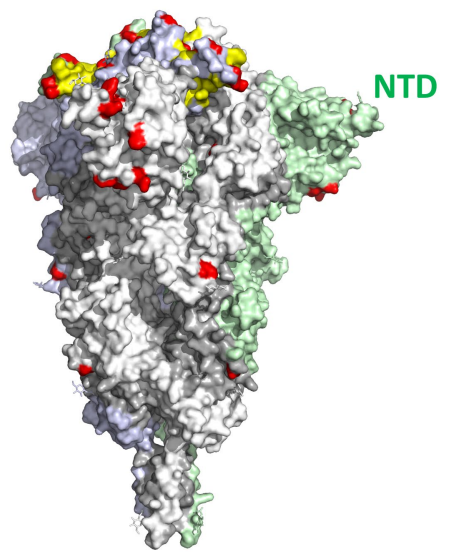

Front subunit removed

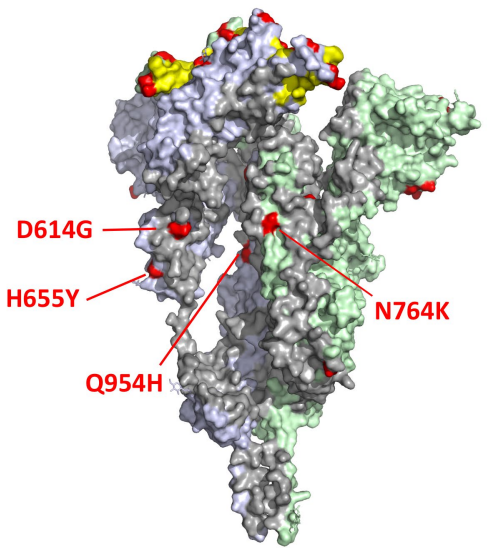

b.
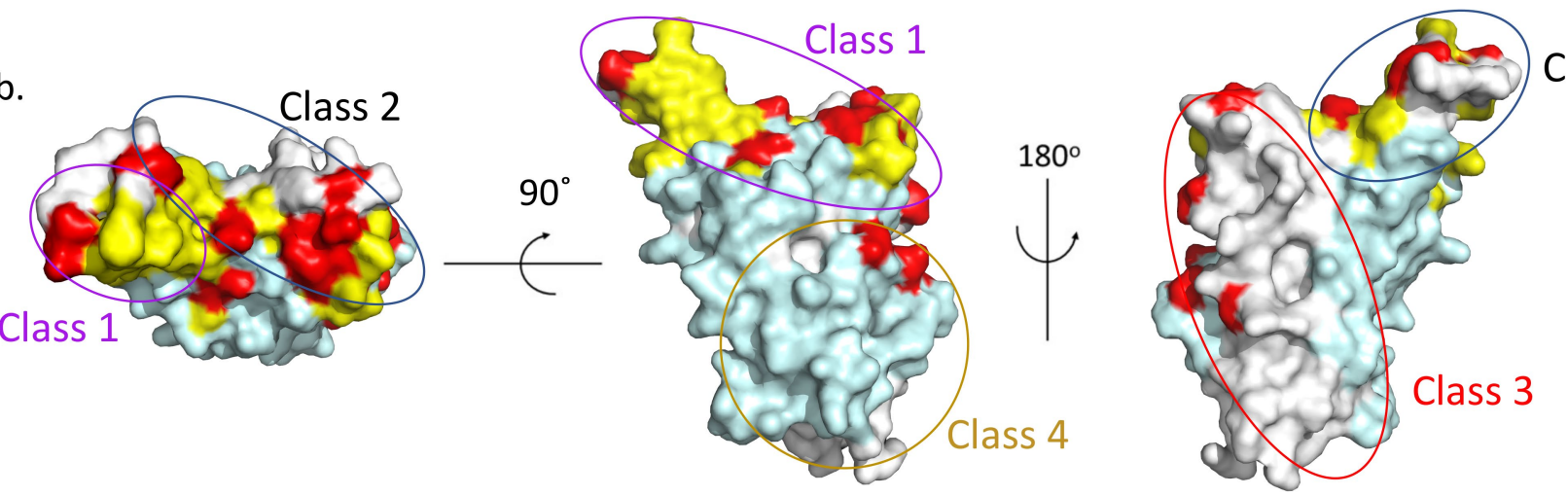

Class 2

Extended Data Fig. 3 | Mapping of the mutations present in Omicron to the spike's surface. a. The spike shown in top (left panel) and in side view (middle and right panels). The spike trimer is shown in surface representation with the three protomers coloured in light grey, light blue and light green. $\mathrm{N}$-terminal and the receptor-binding (NTD and RBD) domains are labelled for the protomer in green only. The represented spike (PDB: 6XR8) is in the closed conformation, i.e., with all three RBDs in the "Down" conformation ${ }^{44}$. The RBD surface of interaction with hACE2 (which is partially occluded in a closed spike) is coloured in yellow. The amino acid differences in the spike of the Omicron variant with respect to the initial Wuhan sequence are marked in red. In the right panel, the front subunit was removed to show changes in S2 and in the C-terminal segment of S1 (labelled) that map to the trimer interface, which could impact the stability of the spike trimer.b. The RBD view down the hACE2 binding surface (left panel) and in two other or thogonal orientations (middle and right panel), as indicated. The hACE2 binding surface is coloured in yellow and the residues altered in Omicron are in red. The RBD surfaces that are buried and exposed in a closed spike are coloured in light cyan and white, respectively. The ovals outline the location of the epitopes of neutralizing antibodies of the various classes that have been described ${ }^{17}$. 
a.
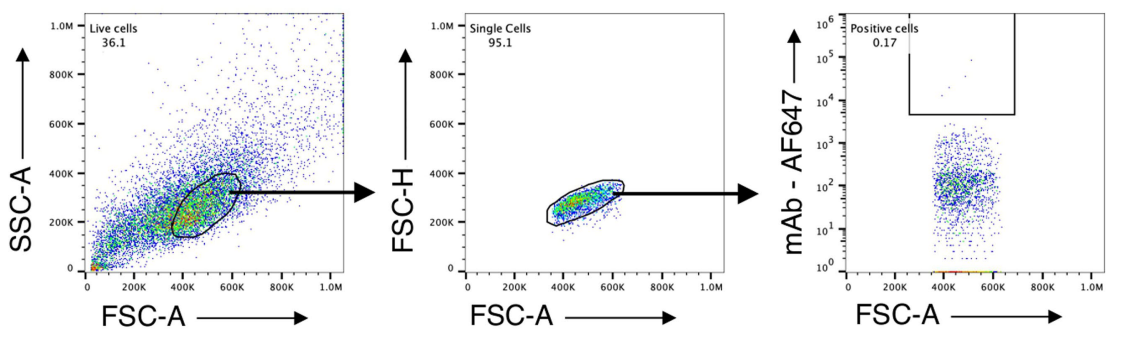

Delta-infected

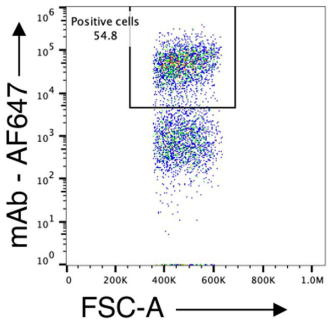

b.

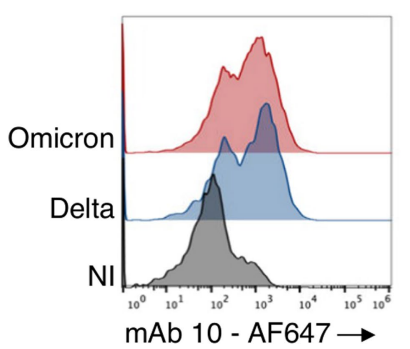

C.

$1 \mu \mathrm{g} / \mathrm{mL}$

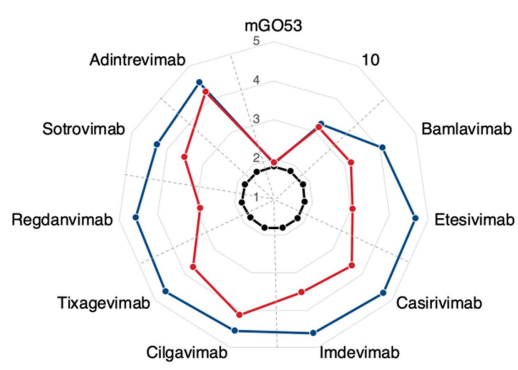

$0.1 \mu \mathrm{g} / \mathrm{mL}$

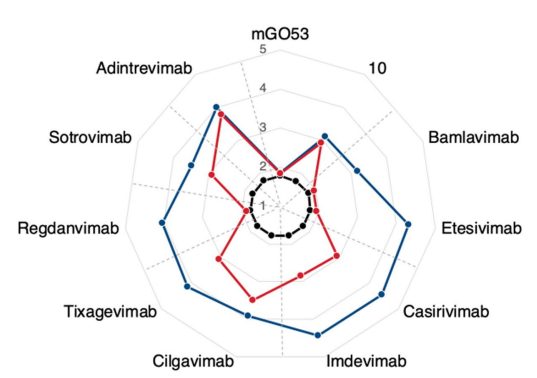

Non-infected

Delta

$\rightarrow$ Omicron

d.

\begin{tabular}{|c|c|c|c|c|c|c|c|}
\hline & & \multirow{2}{*}{\multicolumn{2}{|c|}{ Neutralization (IC50 ng/ml) }} & \multicolumn{4}{|c|}{ Binding (Fold-decrease Omicron/Delta) } \\
\hline & & & & \multicolumn{2}{|c|}{$1 \mu \mathrm{g} / \mathrm{mL}$} & \multicolumn{2}{|c|}{$0.1 \mu \mathrm{g} / \mathrm{mL}$} \\
\hline & & Delta & Omicron & Delta & Omicron & Delta & Omicron \\
\hline \multirow{3}{*}{ Lilly } & Bamlavimab & $>9000$ & $>9000$ & 1 & 8 & 1 & 17 \\
\hline & Etesivimab & 15.4 & $>9000$ & 1 & 42 & 1 & 242 \\
\hline & Bamla/Ete & 20.5 & $>9000$ & 1 & n.d & 1 & n.d \\
\hline \multirow{3}{*}{ Regeneron } & Casirivimab & 3.1 & $>9000$ & 1 & 12 & 1 & 32 \\
\hline & Imdevimab & 6.5 & $>9000$ & 1 & 12 & 1 & 39 \\
\hline & Casirivimab/Imdevimab & 7.6 & $>9000$ & 1 & n.d & 1 & n.d \\
\hline \multirow{3}{*}{ AstraZeneca } & Cilgavimab & 50.3 & 1213 & 1 & 3 & 1 & 3 \\
\hline & Tixagevimab & 18.3 & 8305 & 1 & 9 & 1 & 11 \\
\hline & Cilgavimab/Tixagevimab & 13.4 & 773 & 1 & n.d & 1 & n.d \\
\hline Celltrion & Regdanvimab & 86.6 & $>9000$ & 1 & 47 & 1 & 145 \\
\hline GlaxoSmithKline & Sotrovimab & 325 & 917 & 1 & 6 & 1 & 4 \\
\hline Adagio & Adintrevimab & 19.6 & 403 & 1 & 2 & 1 & 2 \\
\hline
\end{tabular}

Extended Data Fig. 4 | Binding of anti-SARS-CoV-2 monoclonal antibodies to Vero cells infected with Delta and Omicron variants. Vero cells were infected with the indicated variants at an MOI of 0.01 . After $48 \mathrm{~h}$, cells were stained with 1 or $0.1 \mu \mathrm{g} \mathrm{ml}^{-1}$ of the indicated anti-SARS-CoV-2 monoclonal antibodies (Bamlanivimab, Etesevimab, Casirivimab, Imdevimab, Adintrevimab, Cilgavimab, Tixagevimab, Regdanvimab, Sotrovimab) and analysed by flow-cy tometry. a. Gating strategy and example of gates on negative (non-infected) or positive (Delta-infected) samples. b. The anti-S2 pan-coronavirus $\mathrm{mAb} 10$ was used to measure the percentage of infected cells. Histograms show binding of $\mathrm{mAb} 10$ to Vero cells infected with the indicated variants. c. Radar charts represent for each antibody the logarithm of the median fluorescent intensity (MFI) of the staining. Data are representative of two or three independent experiments. d. Inhibitory Concentrations 50\% (IC50) of mAbs against Delta and Omicron variants. The IC50 of the indicated mAbs and some of their combinations were calculated from the neutralization curves displayed in Fig. $1 \mathrm{~b}$. Results are in $\mathrm{ng} \mathrm{ml}^{-1}$. Colour code: Grey: inactive $\mathrm{mAbs}$. Green: $\mathrm{mAbs}$ displaying a neutralizing activity. The binding activity was measured by flow cytometry on Vero cells infected with the indicated variants. Results are presented as the fold-decrease of binding to Omicron-infected cells relative to Delta-infected cells. 
Article

a.

2 doses

Pfizer M5

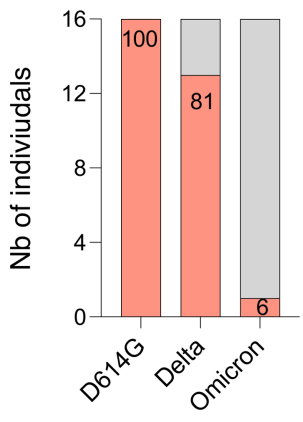

AstraZeneca M5
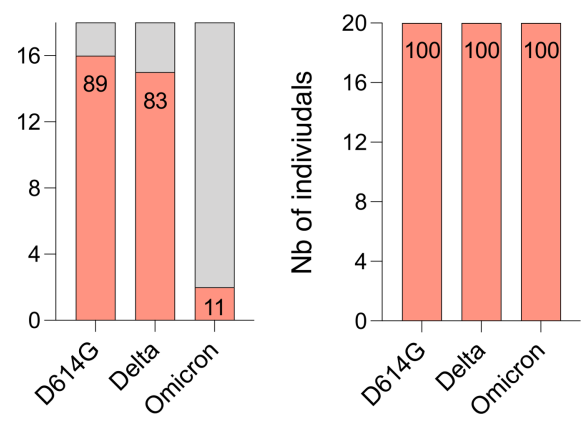

Neutralizer (ED50>30)

Non-neutralizer (ED50<30)

Extended Data Fig. 5 | Fraction of neutralizers in the cohorts of vaccinated or convalescent individuals. Individuals with an ED50 of neutralization above 30 were categorized as neutralizers and are indicated in pink. Non-neutralizers are in grey. The numbers indicate the percentage of neutralizers. $\mathbf{a}$. Fraction of neutralizers in ser from Pfizer $(n=16)$ (left panel) and AstraZeneca $(n=18)$ (right panel) vaccinated recipients sampled 5 months after the second dose (results related to Fig. 2a).b. Fraction of neutralizers in ser from Pfizer

b. 3 doses

Pfizer M1

Convalescents

c.
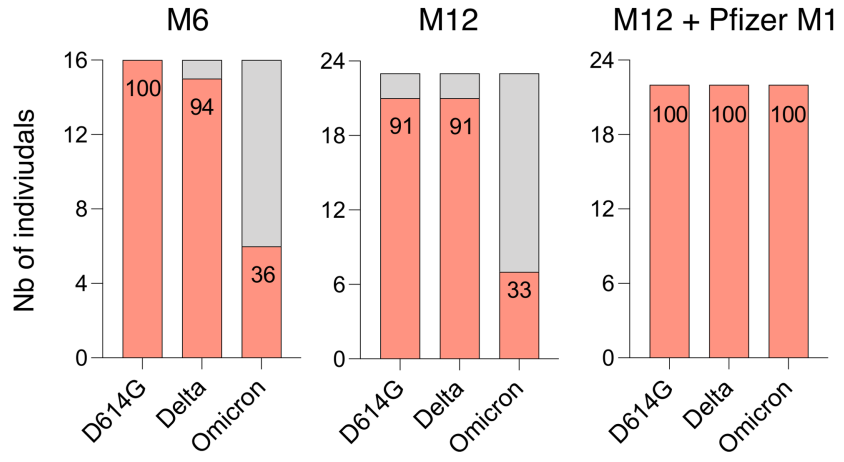

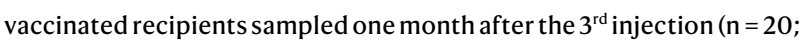
(results related to Fig. 2b).c. Fraction of neutralizers in ser from convalescent individuals, sampled at 6 months post onset of symptoms (M6) $(n=16)$ (right panel), at 12 months (M12) $(n=23)$ (middle panel) and one month after the $1^{\text {st }}$ injection ( $n=22)$ (right panel; results related to Fig. $2 c$ ). In each panel, data are mean from 2 to 3 independent experiments. 
Extended Data Table 1 | Characteristics of the two cohorts of vaccinated and convalescent individuals

a. Vaccinees

$\begin{array}{lll}\begin{array}{l}\text { AstraZeneca M5 } \\ \text { (2 doses) }\end{array} & \begin{array}{l}\text { Pfizer M5 } \\ \text { (2 doses) }\end{array} & \begin{array}{l}\text { Pfizer M1 } \\ \text { (3 doses) }\end{array} \\ \mathrm{n}=18 & \mathrm{n}=16 & \mathrm{n}=\mathbf{2 0}\end{array}$

\begin{tabular}{|c|c|c|c|c|}
\hline \multicolumn{5}{|l|}{ Sex } \\
\hline & Female & 12 & 6 & 9 \\
\hline & Male & 6 & 10 & 11 \\
\hline Age (median; range) & & $60(55 ; 78)$ & $53(33 ; 74)$ & $53(33 ; 74)$ \\
\hline Immune deficiency & & 0 & 0 & 0 \\
\hline Previous COVID-19 & & 0 & 0 & 0 \\
\hline Anti-N & & 0 & 0 & 0 \\
\hline 1st shot & & $\begin{array}{l}\text { Feb } 5 \text { - April 7, } \\
2021\end{array}$ & Jan 6- Feb 4,2021 & Jan 6- April12,2021 \\
\hline 2nd shot & & May3-19, 2021 & $\begin{array}{l}\text { Jan } 28 \text { - March 3, } \\
2021\end{array}$ & $\begin{array}{l}\text { Jan } 28 \text { - May 8, } \\
2021\end{array}$ \\
\hline 3rd shot & & - & - & $\begin{array}{l}\text { July } 1 \text {-Nov 25, } \\
2021\end{array}$ \\
\hline $\begin{array}{l}\text { Sampling days post- } \\
2^{\text {nd }} \text { dose; M5 (median; } \\
\text { range) }\end{array}$ & & $\begin{array}{l}150 \\
(110-178)\end{array}$ & $\begin{array}{l}161 \\
(138-176)\end{array}$ & - \\
\hline $\begin{array}{l}\text { Sampling days post- } \\
3^{\text {rd }} \text { dose; M1 (median; } \\
\text { range) }\end{array}$ & & - & - & $\begin{array}{l}33 \\
(8-61)\end{array}$ \\
\hline
\end{tabular}

b. Convalescents

$6 \mathrm{M}$ POS 12M POS 12M POS

$n=16$ then vaccinated

\begin{tabular}{|c|c|c|c|c|}
\hline \multicolumn{5}{|l|}{ Sex } \\
\hline & Female & 10 & 11 & 11 \\
\hline & Male & 6 & 12 & 11 \\
\hline Age (Median; range) & & $56(32 ; 77)$ & $52(23 ; 82)$ & $52(23 ; 82)$ \\
\hline \multicolumn{5}{|l|}{ Severity } \\
\hline & Severe & 8 & 13 & 9 \\
\hline & Mild-Moderate & 7 & 8 & 4 \\
\hline & Asymptomatic & 1 & 2 & 1 \\
\hline PCR & & 15 & 21 & 20 \\
\hline Anti-S (S-Flow) & & 16 & 23 & 22 \\
\hline $\begin{array}{l}\text { Sampling days POS (median; } \\
\text { range) }\end{array}$ & & $\begin{array}{l}184 \\
(129 ; 195)\end{array}$ & $\begin{array}{l}368 \\
(344-454)\end{array}$ & - \\
\hline $\begin{array}{l}\text { Days between the vaccination } \\
\text { and onset of symptoms (median; } \\
\text { range) }\end{array}$ & & - & - & $\begin{array}{l}394 \\
(353-444)\end{array}$ \\
\hline $\begin{array}{l}\text { Sampling days post-vaccination } \\
\text { (median; range) }\end{array}$ & & - & - & $\begin{array}{l}32 \\
(21-48)\end{array}$ \\
\hline
\end{tabular}




\section{Reporting Summary}

Nature Research wishes to improve the reproducibility of the work that we publish. This form provides structure for consistency and transparency in reporting. For further information on Nature Research policies, see our Editorial Policies and the Editorial Policy Checklist.

\section{Statistics}

For all statistical analyses, confirm that the following items are present in the figure legend, table legend, main text, or Methods section.

n/a Confirmed

$\square$ The exact sample size $(n)$ for each experimental group/condition, given as a discrete number and unit of measurement

$\square$ \A statement on whether measurements were taken from distinct samples or whether the same sample was measured repeatedly

$\square$ The statistical test(s) used AND whether they are one- or two-sided

$\square$ Only common tests should be described solely by name; describe more complex techniques in the Methods section.

$\square$ A description of all covariates tested

$\square$ \A description of any assumptions or corrections, such as tests of normality and adjustment for multiple comparisons

$\square$ A full description of the statistical parameters including central tendency (e.g. means) or other basic estimates (e.g. regression coefficient)

$\triangle$ AND variation (e.g. standard deviation) or associated estimates of uncertainty (e.g. confidence intervals)

For null hypothesis testing, the test statistic (e.g. $F, t, r$ ) with confidence intervals, effect sizes, degrees of freedom and $P$ value noted

Give $P$ values as exact values whenever suitable.

Х $\square$ For Bayesian analysis, information on the choice of priors and Markov chain Monte Carlo settings

Х $\square$ For hierarchical and complex designs, identification of the appropriate level for tests and full reporting of outcomes

Х $\square$ Estimates of effect sizes (e.g. Cohen's $d$, Pearson's $r$ ), indicating how they were calculated

Our web collection on statistics for biologists contains articles on many of the points above.

\section{Software and code}

Policy information about availability of computer code

Data collection Harmony Software v4.9 (Perkin-Elmer), Attune Nxt Software v3.2.1 (ThermoFischer), Flowjo Software v10.7.1

Data analysis Excel 365 v16.46 (Microsoft), Prism v9.0.2 (GraphPad Software)

For manuscripts utilizing custom algorithms or software that are central to the research but not yet described in published literature, software must be made available to editors and reviewers. We strongly encourage code deposition in a community repository (e.g. GitHub). See the Nature Research guidelines for submitting code \& software for further information.

\section{Data}

Policy information about availability of data

All manuscripts must include a data availability statement. This statement should provide the following information, where applicable:

- Accession codes, unique identifiers, or web links for publicly available datasets

- A list of figures that have associated raw data

- A description of any restrictions on data availability

All data associated with this study are available from O.S 


\section{Field-specific reporting}

Please select the one below that is the best fit for your research. If you are not sure, read the appropriate sections before making your selection.

$\bigotimes$ Life sciences $\quad \square$ Behavioural \& social sciences $\quad \square$ Ecological, evolutionary \& environmental sciences

For a reference copy of the document with all sections, see nature.com/documents/nr-reporting-summary-flat.pdf

\section{Life sciences study design}

All studies must disclose on these points even when the disclosure is negative.

Sample size $\quad 131$ sera from convalescent, vaccinated and vaccinated convalescent individuals were analyzed in the study. Given the explanatory nature of the study aiming at describing a phenomenon whose frequency has not yet been established it was not possible to use statistical methods were used to predetermine sample size. Thus, we included between 10 and 50 patients per group.

Data exclusions None.

Replication All experiments were performed and verified in multiple replicates as indicated in their methods/figure legends.

Randomization The experiments were not randomized as we tested all available samples.

Blinding The investigators were not blinded to allocation during experiments and outcome assessment. However, the clinical sampling and biological measurement were performed by different teams. Only the final assembly of the data revealed the global view of the results.

\section{Reporting for specific materials, systems and methods}

We require information from authors about some types of materials, experimental systems and methods used in many studies. Here, indicate whether each material, system or method listed is relevant to your study. If you are not sure if a list item applies to your research, read the appropriate section before selecting a response.

Materials \& experimental systems

$\mathrm{n} / \mathrm{a}$ Involved in the study

$\square$ Antibodies

$\square \bigotimes$ Eukaryotic cell lines

Х Palaeontology and archaeology

\ $\square$ Animals and other organisms

$\square$ Wuman research participants

$\square$ Clinical data

X $\square$ Dual use research of concern

\begin{tabular}{l} 
Methods \\
\hline$n / a$ \\
$\square$
\end{tabular}

Antibodies

Antibodies used

Adintrevimab, Cilgavimab, Regdavimab, Sotrovimab and Tixagevimab are human anti-S monoclonal antibodies produced by Hugo Mouquet (Institut Pasteur). Bamlanivimab, Etesivimab, Casirivimab and Imdevimab were kind gifts of Thierry Prazuck and Laurent Hocqueloux. The Goat anti-Human IgG $(\mathrm{H}+\mathrm{L})$ Cross-Adsorbed Secondary Antibody, Alexa Fluor 647 (A21445) was obtained from thermoFisher Scientific.

Validation

Adintrevimab, Cilgavimab, Regdavimab, Sotrovimab and Tixagevimab were validated using ELISA binding assays (against the trimeric S, RBD, and S2 proteins) by the team of H.Mouquet. Bamlanivimab, Etesivimab, Casirivimab and Imdevimab were validated by measuring their neutralizing activity against SARS-CoV-2. Validation of the goat anti-human IgG is available from the ThermoFisher website.

\section{Eukaryotic cell lines}

Policy information about cell lines

Cell line source(s)

Authentication

Mycoplasma contamination
Vero E6 (ATCC ${ }^{\circledast}$ CRL-1586 ${ }^{\mathrm{TM}}$ ), 293T cells (ATCC CRL- 3216), Freestyle 293-F (ThermoFisher) and U2OS cells (ATCC ${ }^{\circledast}$ HTB-96 ${ }^{\mathrm{TM}}$ ), all obtained from the ATCC.

Cell lines were not authenticated.

All cells are negative for mycoplasma contamination. Tests are performed every Monday. 
Commonly misidentified lines

(See ICLAC register)

\section{Human research participants}

\section{Policy information about studies involving human research participants}

Population characteristics Orleans' Cohort of convalescent and/or vaccinated individuals: since April 2020, a prospective, monocentric, longitudinal, cohort clinical study enrolling 170 SARS-CoV-2-infected individuals and 30 non-infected healthy controls is on-going, aiming to describe the persistence of specific and neutralizing antibodies over a 24-months period.

Given the exploratory design of the study, the characteristics of participants were not pre-established when entering the cohorts. Relevant co-variates are provided in the corresponding supplementary tables.

Recruitment

Orléans cohort : Individuals admitted to the hospital for COVID-19 vaccination, COVID-19 or with known COVID-19 consulting for a chronic disease were invited to participate.

Individuals were included without any selection other than those imposed by the entry criteria (known COVID-19 or vaccination). Under these conditions, no particular bias is envisaged.

Ethics oversight

Orléans was approved by national external committee (CPP Ile de France IV, IRB No. 00003835). At enrolment a written informed consent was collected for all participants.

Note that full information on the approval of the study protocol must also be provided in the manuscript.

\section{Clinical data}

Policy information about clinical studies

All manuscripts should comply with the ICMJE guidelines for publication of clinical research and a completed CONSORT checklist must be included with all submissions.

Clinical trial registration NCT04750720

Study protocol

All protocols can be accessed on clinicaltrial.gov

Data collection

The cohort started on April 2020 in Orléans Hospital (Centre hospitalier Réginal Orléans), and is on-going.

Outcomes

The primary outcome of the study was the presence of antibody to SARS-CoV-2 antibody binding to the spike protein (S-Flow assay). The secondary outcome of the assay was the presence of neutralizing antibodies (S-Fuse assay)

\section{Flow Cytometry}

Plots

Confirm that:

$\bigotimes$ The axis labels state the marker and fluorochrome used (e.g. CD4-FITC).

\The axis scales are clearly visible. Include numbers along axes only for bottom left plot of group (a 'group' is an analysis of identical markers).

$\bigotimes$ All plots are contour plots with outliers or pseudocolor plots.

$\bigotimes$ A numerical value for number of cells or percentage (with statistics) is provided.

\section{Methodology}

Sample preparation

Instrument

Software

Cell population abundance

Gating strategy
SARS-CoV-2 infected Vero cells were stained as indicated in the method section. All samples were acquired within $24 \mathrm{~h}$.

Attune NxT Acoustic Focusing Cytometer, blue/red/violet/yellow (catalog number : 15360667)

AttuneNxT Software v3.2.1

At least 10,000 cells were acquired for each condition.

All gates were set on uninfected Vero cells.

$\bigotimes$ Tick this box to confirm that a figure exemplifying the gating strategy is provided in the Supplementary Information. 